\title{
Proportions. Arts, architecture, musique, mathématiques et sciences
}

Tours, du 30 juin au 4 juillet 2008

\section{Sabine Rommevaux, Philippe Vendrix et Vasco Zara}

\section{(2) OpenEdition}

\section{Journals}

Édition électronique

URL : https://journals.openedition.org/cem/7302

DOI : $10.4000 /$ cem.7302

ISSN : 1954-3093

Éditeur

Centre d'études médiévales Saint-Germain d'Auxerre

Édition imprimée

Date de publication : 15 août 2008

ISSN : 1623-5770

\section{Référence électronique}

Sabine Rommevaux, Philippe Vendrix et Vasco Zara, «Proportions. Arts, architecture, musique,

mathématiques et sciences ", Bulletin du centre d'études médiévales d'Auxerre | BUCEMA [En ligne], 12 |

2008, mis en ligne le 09 avril 2009, consulté le 22 septembre 2022. URL : http://

journals.openedition.org/cem/7302 ; DOI : https://doi.org/10.4000/cem.7302

Ce document a été généré automatiquement le 22 septembre 2022.

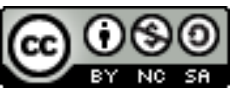

Creative Commons - Attribution - Pas d'Utilisation Commerciale - Partage dans les Mêmes Conditions 4.0 International - CC BY-NC-SA 4.0

https://creativecommons.org/licenses/by-nc-sa/4.0/ 


\section{Proportions. Arts, architecture, musique, mathématiques et sciences}

Tours, du 30 juin au 4 juillet 2008

Sabine Rommevaux, Philippe Vendrix et Vasco Zara

1 Le langage des proportions est le langage dans lequel s'écrivent les mathématiques mais aussi leurs applications, depuis l'Antiquité jusqu'au XVIIe siècle, jusqu'à ce que le langage des fonctions vienne le supplanter. Ainsi, par exemple, durant toute cette longue période, il n'est pas dit que la circonférence d'un cercle s'exprime en fonction du rayon de ce cercle et encore moins que cette circonférence vaille ce rayon multiplié par $2 \pi$, mais il est dit que les circonférences des cercles sont proportionnelles à leurs rayons. Il est dit, de même, que les vitesses des mobiles sont proportionnelles au temps, ou inversement proportionnelles aux distances parcourues; ou encore que la hauteur d'un son est proportionnelle à la longueur de la corde que l'on pince. Ce faisant, toute propriété mathématique ou physique est exprimée sur le mode de la comparaison: on compare deux cercles, deux mouvements, deux sons, etc. Mais le langage des proportions n'est pas restreint au seul champ des mathématiques et de ses applications. Le principe de comparaison qui le sous-tend peut être étendu en un principe philosophique plus général d'analogie, voire en un principe d'harmonie. On sait l'importance que jouent ces principes dans les philosophies néo-platoniciennes, notamment. S'intéresser aux proportions, c'est donc s'intéresser à tous les domaines du savoir, scientifiques ou philosophiques.

Dans ce colloque ${ }^{1}$, nous souhaitons aborder tous les aspects de la proportion. Nous l'aborderons donc en tant qu'objet de théories mathématiques, en tant qu'outil dans tous les domaines du savoir et en tant que principe au cœur de constructions épistémologiques ou philosophiques. Nous couvrirons la période allant du XIV siècle au $\mathrm{XVII}^{\mathrm{e}}$ siècle. Après le $\mathrm{xvII}^{\mathrm{e}}$ siècle, si les principes philosophiques d'analogie ou d'harmonie perdurent, la théorie mathématique des proportions devient obsolète. Au $\mathrm{XIV}^{\mathrm{e}}$ siècle, on voit se développer de nouvelles théories mathématiques des proportions, suscitées par de nombreuses applications, parfois nouvelles. Ainsi, à Oxford, dans le cercle de ceux que l'on nommera les Calculatores, l'application de la théorie des 
proportions à l'étude du mouvement, dans le cadre des commentaires à Aristote, débouche sur la notion non euclidienne de rapport entre des rapports et se met en place à cette occasion une théorie qui s'ancre à la fois dans les Éléments d'Euclide et dans l'Arithmétique de Nicomaque, transmise au monde latin par Boèce. Dans le sillage de ces interprétations des traités d'Aristote apparaît, dans le domaine musical, une nouvelle conception des valeurs temporelles du son, comme en témoigne le mouvement de l'Ars nova, tant dans la notation que dans la composition. De même, c'est à cette époque que commence l'extraordinaire exégèse vitruvienne, qui, actualisant la conception de la proportion, fera de l'architecture à la Renaissance la pierre angulaire des savoirs artistiques.

3 Nous examinerons donc ces nouvelles théories, en montrant comment elles dépassent la théorie euclidienne en s'appuyant sur d'autres textes que les Éléments, mais aussi comment elles prennent en compte les exigences posées par leurs applications. Nous verrons aussi si l'émergence de ces nouvelles théories a des répercussions dans le champ de la philosophie ou de l'épistémologie.

4 Le colloque s'articulera autour de sept demi-journées. Trois demi-journées seront centrées autour d'une discipline, puisque les proportions jouent un rôle fondateur dans trois domaines: l'algèbre, la musique et l'architecture. Quatre demi-journées proposeront une approche pluridisciplinaire et auront pour thème: arts et proportions, mystères et proportions, harmonie et sciences, proportions en théorie et en pratique.

\section{Algèbre et proportion}

5 À la fin de la Renaissance et au début de l'Âge classique, on sait que la théorie des proportions reste encore un instrument privilégié pour la résolution de nombreux problèmes d'algèbre. La théorie des proportions offre aussi parfois un critère de validité des résultats que l'on peut trouver par ailleurs grâce à un raisonnement de type algébrique. Durant cette demi-journée, nous poserons donc la question du rôle joué par la théorie des proportions dans la constitution de l'algèbre durant cette période. Et ce faisant, nous serons amenés à nous interroger sur la nature de la théorie des proportions qui est ici en jeu. S'agit-il de la théorie euclidienne? Son usage dans le cadre de problèmes algébriques l'a-t-elle modifiée, a-t-elle conduit à de nouveaux concepts?

\section{Arts et proportions}

6 Outils mathématiques, le concept de proportion déploie tout son potentiel pratique en devenant langage constructeur d'harmonie. La théorie des proportions, qui est la science du rapport et non celle du nombre, s'ouvre sur un vaste champ de relations épistémologiques qui définissent canon et règle de beauté dans les diverses disciplines artistiques. Pendant cette demi-journée, on s'interrogera donc, à partir de cas exemplaires et paradigmatiques, sur la façon dont, au fil des siècles, chaque savoir technique - en peinture, en musique, en littérature - théorise son propre jugement esthétique sur la proportion, pour, ensuite, le décliner dans la réalisation pratique de son propre objet artistique. 


\section{Proportions et notations musicales}

7 L'introduction des signes de proportion marque un changement radical dans la manière de noter la musique. Si le principe était déjà en germe dans la notation de l'Ars nova, c'est à partir de l'Ars subtilior, dans les deux dernières décennies du XIV ${ }^{\mathrm{e}}$ siècle, que leur emploi apparaît de façon évidente. La notation dite proportionnelle connaît aux $\mathrm{XV}^{\mathrm{e}}$ et $\mathrm{XVI}^{\mathrm{e}}$ siècles une histoire riche : de l'affirmation d'une complexité radicale à une tendance à la simplification afin d'abandonner le système relatif au profit d'un système absolu - le système binaire de la notation moderne. Si cette histoire est aujourd'hui relativement bien connue, quelques énigmes demeurent: comment mesurer l'impact de cette notation proportionnelle sur la manière de composer et d'interpréter la musique des $\mathrm{XV}^{\mathrm{e}}$ et $\mathrm{XVI}^{\mathrm{e}}$ siècles ? Quelles relations les théoriciens de la notation entretiennent-ils avec les savants spécialistes de proportion? Dans quelles conditions s'est opéré le divorce entre les principes de notation et leur applicabilité pour les compositeurs?

\section{Mystères et proportions}

8 Sans doute est-ce l'observation d'un phénomène simple qui est à l'origine d'une des traditions théoriques les plus vivaces jusqu'à la fin de la Renaissance : lorsque des corps se heurtent, ils produisent des sons. De ce constat, des «Pythagoriciens » en déduisent que les sphères célestes, des corps énormes, qui bougent à des vitesses différentes, produisent certainement des sons incroyables. Ces sons sont toutefois susceptibles d'être mesurés. Car la vitesse des sphères, calculée à partir de leur distance, démontre une organisation proportionnelle semblable aux accords musicaux, et cette similitude donne à penser que les sons produits par les planètes obéissent également à un système proportionnel, dont le fonctionnement procède des mêmes lois que celles qui régissent les harmonies musicales. Plaisir, analogie, création artistique et observation scientifique se croisent et se manifestent sous des formes diverses, parfois difficiles à concilier. Il s'agira durant cette session de confronter des modes d'approche, des expériences, scientifiques ou artistiques, qui débouchent sur des textes ou sur des œuvres. Comment tisser des liens entre les canons proportionnels qu'explorent avec virtuosité les compositeurs du début du Xvi ${ }^{\mathrm{e}}$ siècle et les harmonies célestes de Kepler ? La découverte de la solution des canons musicaux - excessivement difficile dans certains cas - et le recours au principe de proportion dans les traités d'alchimie se construisent-ils de manière similaire?

\section{Architecture}

C'est à partir d'Alberti que l'architecte, soucieux de théoriser son savoir sur le modèle vitruvien, se penche à nouveau sur les principes fondant sa discipline. De l'humanisme albertien aux méta-projets palladiens, en passant par le florilège encyclopédique du $\mathrm{XVI}^{\mathrm{e}}$ siècle, l'histoire est apparemment bien connue, comme le démontrent différentes lectures critiques publiées ces dernières années. Si le cadre interprétatif dépasse souvent le seul savoir architectural, est alors venu le moment d'en éclairer tous les passages et les problématiques concernés, à partir d'un nouvel examen critique des 
notions fondatrices, tels que la proportionnalité et les ordres: quel changement entraîne la relecture philologique des différents commentaires au De Architectura commencée au $\mathrm{xv}^{\mathrm{e}}$ siècle? De quelle façon la théorie des ordres architecturaux, expression du canon mathématique, arrive t-elle à concilier anthropomorphisme et expression des passions? Que devient la notion d'harmonie engendrée par la définition de la proportion sous l'impact des querelles artistiques au XVII ${ }^{\mathrm{e}}$ siècle?

\section{Harmonie et sciences}

10 Le principe philosophique d'harmonie de même que celui d'analogie sont au cœur de certaines constructions scientifiques que nous souhaitons examiner lors de cette demijournée. Nous verrons de quelle façon ces principes aident à la compréhension des phénomènes de la nature. Est-ce que ces principes apparaissent dans les théories scientifiques à la suite de la contemplation de ces phénomènes, ou, en d'autres termes, est-ce que les savants croient les reconnaître dans la nature? Ou bien, est-ce que ces principes régissent a priori la pensée scientifique formant en quelque sorte le regard que portent les savants sur les phénomènes qu'ils souhaitent expliquer?

\section{Proportions entre pratique et théorie}

Lors de cette session, nous poserons de manière plus spécifique la question de l'émergence de nouvelles théories des proportions entre le XIV siècle et le XVII ${ }^{\mathrm{e}}$ siècle. Nous nous demanderons en particulier si l'essor de l'utilisation de la proportionnalité dans de nombreux domaines très divers - essor particulièrement important durant cette période - entraîne des réflexions nouvelles sur les objets et les principes de la théorie des proportions, si elle engendre de nouveaux résultats. C'est ainsi, l'articulation entre pratique et théorie qui sera au cœur de cette demi-journée.

\section{Participants :}

- Mohammed Abattouy

- Mohammed Abattouy

- Valérie Auclair

- Anna Marie Busse Berger

- Filippo Camerota

- Mario Carpo

- Pierre Caye

- Thomas Cristensen

- Jehanne Dautrey

- Jean Dhombres

-J. V. Fields

- Jens Hoyrup

- Gabriela Ilnitchi

- Bernard Joly

- Matthew Landrus

- Henrique Leitao

- Frédérique Lemerle 
- Stefano Lorenzetti

- Antoni Malet

- Guido Mambella

- Jean-Claude Margolin

- Laura Moretti

- Ann E. Moyer

- Yves Pauwels

- David Rabouin

- Rudolf Rasch

- Pietro Roccasecca

- Sabine Rommevaux

- Danilo Samsa

- Katelijne Schiltz

- Gérard Simon

- Maryvonne Spiesser

- Ann Stone

- Dorit Tanay

- Philippe Vendrix

- Lucien Vinciguerra

- Rob Wegman

- Brigitte van Wymersch

- Vasco Zara

- Frank Zöllner

\section{NOTES}

1. Avec la collaboration de l'UMR 5594 ARTeHIS ; Artes Renascentes. Centre d'études supérieures de la Renaissance, UMR 6576, CNRS, université François-Rabelais, Tours, LI $^{\mathrm{e}}$ colloque d'études humanistes, « http://www.cesr.univ-tours.fr ».

INDEX

Mots-clés : architecture, musique, proportion 